\title{
ABHAY KISAAN: Aiding Farmers with AI
}

\author{
Aakash Barhate \\ INFT Department VESIT \\ Mumbai, India \\ 2017.aakash.barhate@ves.ac.in \\ Dr.Shalu Chopra \\ INFT Department VESIT \\ Mumbai, India \\ shalu.chopra@ves.ac.in
}

\author{
Atharva Potdar \\ INFT Department VESIT \\ Mumbai, India \\ 2017.atharva.potdar@ves.ac.in
}

\author{
Vedant Sonje \\ INFT Department VESIT \\ Mumbai, India \\ 2017.vedant.sonje@ves.ac.in
}

\begin{abstract}
Abhay Kisaan is a project aimed at protecting the integrity of farmers by developing three modules for their benevolence. The first module is a crop recommender system which recommends crops based on climate and soil conditions. The second module is a weed detection system which detects the exact location and presence of weed in an image of a top view crop field. Weed growth can hamper crop development and hence must be detected and removed at once. The third module is Prediction of various crop diseases using images and analyzing its cause to present it as evidence for a faulty fertilizer or inclement weather. Accuracy of the models are 95 will be presented as android application.
\end{abstract}

Index Terms-Tensorflow, Scikit-learn, Convolutional Neural Network (CNN), Object-Detection, FRCNN V2,weed, crop, accu- racy.

\section{INTRODUCTION}

India is one of the established nations that has agriculture as its primary source of income. Agriculture is one such domain that contributes only around 14on the Indian economy. The conventional agricultural practices and techniques are posing a lot of issues in terms of efficiency, cost-effectiveness and resource utilization. There is a necessity of better techniques that can improve the standard of living of the farmers too. Over the years due to globalization, agriculture has evolved by adapting the latest technologies and techniques for a better standard of living. Among the technologies and techniques, precision agriculture is one budding technology in the field of agriculture. Precision agriculture mainly focuses on sitespecific farming The major and serious setback in the crop productivity is that the farmers do not choose the right crop for cultivation. There are many different issues regarding farming that still need to be resolved because they create a major impact on agriculture. These issues need to be solved efficiently so that productivity will increase and that will be beneficial for farmers.

Many farmers generally follow their traditional approach towards farming that sometimes ended by choosing the wrong type of crop for farming. Traditional farming is not always effective and it does not lead to proper choice of the crop for farming. Due to extreme weather changes and disturbance of the seasonal weather, particular crops may not be the right choice. This mainly happens because the type of crop for farming is not suitable according to conditions. Particular crops for the farming should be chosen on basis of the weather conditions, location of the farm, ph value of the soil and other factors. These all factors are not considered mainly so that choice of the crop is not proper. Many times crop affected by the particular disease are not identified by farmers and that leads to more destruction of the crop. Due to various factors the crop gets affected by various diseases and not all diseases are common to farmers and hence they face difficulty to identify the diseases. If a particular disease is not identified in the meantime there will be more destruction of the crop. High Percentage of weed on the farm leads to less productivity in the farm, hence to detect the weed in the farm is a challenge for the farmers. Weed is the mostly unwanted part in the farm and over excess of that reduces the productivity of the crop. Since it is difficult to identify the weed farmers face this issue many times and hence there is a need for a solution for this problem.

\section{OBJECTIVES}

The Following are the main objectives :

- Recommendation of multiple crops to farmers according to various parameters.

- Detection of weed in the crop field.

- Disease identification of different crops.

\section{LITERATURE SURVEY}

In the paper S. Pudumular have finalized features for the crop recommendation system but their model generated is prone to overfitting [1]. Author Rohit Rajak has combined datasets from different geographical regions and has performed evaluation of feed forward networks but has limited the use of ensembing [2]. T Hariharan's paper provides insights about evaluation of dataset but the use of Image Segmentation seems irrelevant [3]. It was important to select an appropriate model for weed detection among the likes of FRCNN, YOLO, SSD and gather information about implementation of the weed detection system [4]. The paper is used for getting an idea of how the final module will be implemented with a seamless application [5].

\section{DATASET DESCRIPTION}

For the crop recommendation system, the dataset consists of 4 features namely Temperature, Humidity, Soil $\mathrm{pH}$ value and Rainfall. There are a total of 31 classes including crops like Rice, wheat, maize etc. and the size of the dataset is over 3100 rows. For Crop Disease Identification, the dataset consists of images of 38 classes of crop diseases and the dataset size is close to 50,000 images. For the weed detection module the image dataset of 100 unlabelled images of weed in crop fields are first labelled using LabelImg. The labelled info is then converted from xml to csv. The csv information 
is again converted into a tf records file. After this procedure training of the model is to be done.

\begin{tabular}{|c|c|c|c|c|}
\hline temperature & humidity & ph & rainfall & label \\
\hline 20.87974371 & 82.00274423 & 6.502985292 & 202.9355362 & nce \\
\hline 21.77046169 & 80.31964408 & 7.038096361 & 226.6555374 & rice \\
\hline 23.00445915 & 82.3207629 & 7.840207144 & 263.9642476 & nice \\
\hline 26.49109635 & 80.15836264 & 6.980400905 & 242.8640342 & nce \\
\hline 20.13017482 & 81.60487287 & 7.628472891 & 262.7173405 & nce \\
\hline 23.05804872 & 83.37011772 & 7.073453503 & 251.0549998 & rice \\
\hline 22.70883798 & 82.63941394 & 5.70080568 & 271.3248604 & nice \\
\hline 20.27774362 & 82.89408619 & 5.718627178 & 241.9741949 & nice \\
\hline 24.51588066 & 83.5352163 & 6.685346424 & 230.4462359 & noe \\
\hline 23.22397386 & 83.03322691 & 6.336253525 & 221.2091958 & rice \\
\hline 26.52723513 & 81.41753846 & 5.386167788 & 264.6148697 & nice \\
\hline 23.97898217 & 81.45061596 & 7.50283396 & 250.0832336 & nce \\
\hline
\end{tabular}

Fig. 1. Crop Recommendation System Dataset

\section{PROPOSED SYSTEM}

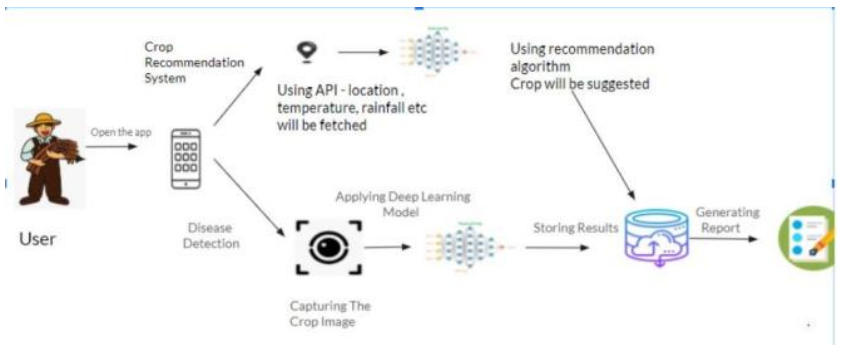

Fig. 2. The proposed system for integrated modules of crop recommender and crop disease prediction

\section{A. Crop Recommendation System}

This module will be presented as an android application. The feature values for prediction will be taken by gaining the user's gps coordinates and then scraping details according to these coordinates.

\section{B. Weed Detection System}

All the top view images of the crop field taken using drones or a camera will be stitched together and the algorithm will be applied on every image. In output the detected images will be stitched similarly. Due to this the user can find out where exactly the weed is.

\section{Crop Disease Prediction System}

This module will also be presented as an android application. The user will then upload the image of the infected crop/leaf. With the help of Image Recognition the exact disease will be predicted. Also the user will know about the cause of this disease by comparing similar information in the database.

\section{ALGORITHMS USED}

\section{A. Crop Recommendation System}

\begin{tabular}{|c|c|}
\hline Algorithms & Accuracy ( In Percentage) \\
\hline 1. NaiveBayes(GaussianNB) & 93.9 \\
2. SVM & 83.3 \\
3. Random Forest & 95.5 \\
4. XGBoost & 94.08 \\
5. Decision Tree & 92.3 \\
6. Voting Classifier & 95.4 \\
7. Stacking Classifier & 95.4 \\
\hline
\end{tabular}

Fig. 3. Algorithms and their accuracy (In Percentage
From the above table we can infer that the ensembling tech- niques like Random Forest, Voting and Stacking Classifiers yield the highest accuracy

\section{B. Weed Detection System}

The technique used for detecting weed in crop fields is Object-Detection. It is implemented using Tensorflow 1.15 along with an Object-Detection directory containing necessary files. The model used for actual detection is Faster RCNN Inception V2. The IoU score goes as high as 0.98 . The no. of boxes to be detected is kept 1 and threshold is kept 0.85

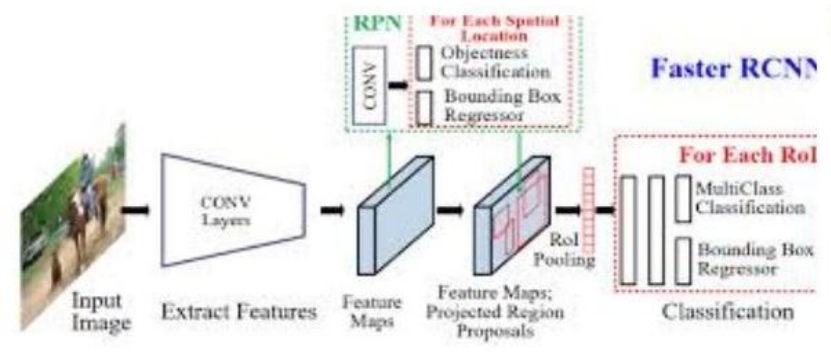

Fig. 4. Image explaining the architecture of Faster RCNN

\section{Crop Disease Prediction}

Used Convolutional Neural Network (CNN) and transfer learning network called Resnet50. Transfer Learning plays a significant role in boosting the accuracy of the model since the no. of classes are 38 and the dataset size is over 50,000 images.

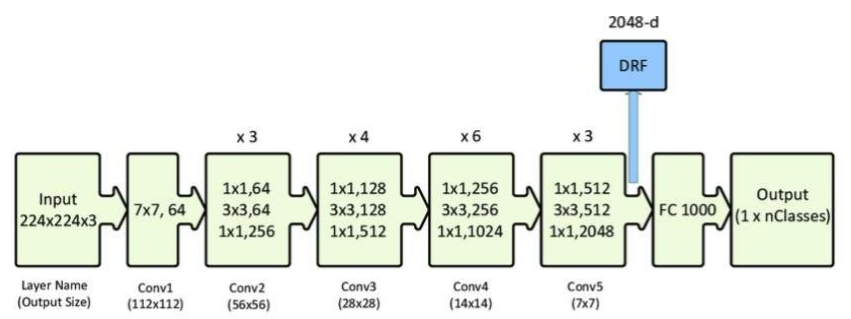

Fig. 5. Architecture of Resnet50

\section{RESULTS}

\section{A. Crop Recommendation System}

According to Fig 3, since Random Forest technique gives the highest accuracy and being an ensembling technique it is preferred over all other techniques [7].

\section{B. Weed Detection System}

The weed detection along with the accuracy percentage is obtained as results

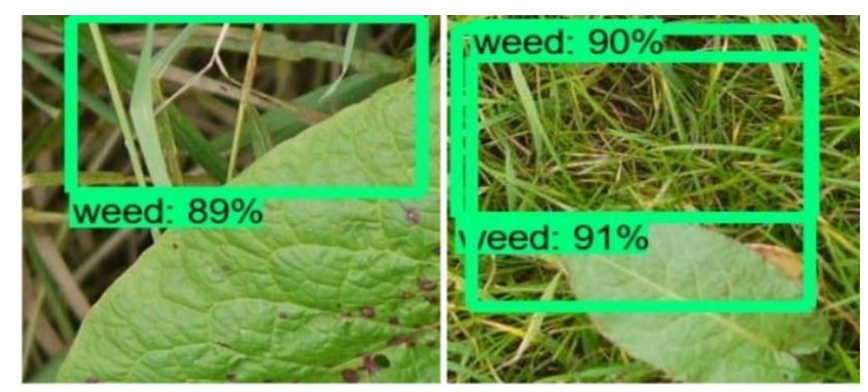

Fig. 6. Weed is detected in above top view images with very high IoU scores 


\section{Crop Disease Prediction}

The accuracy obtained with Resnet50 is close to 91

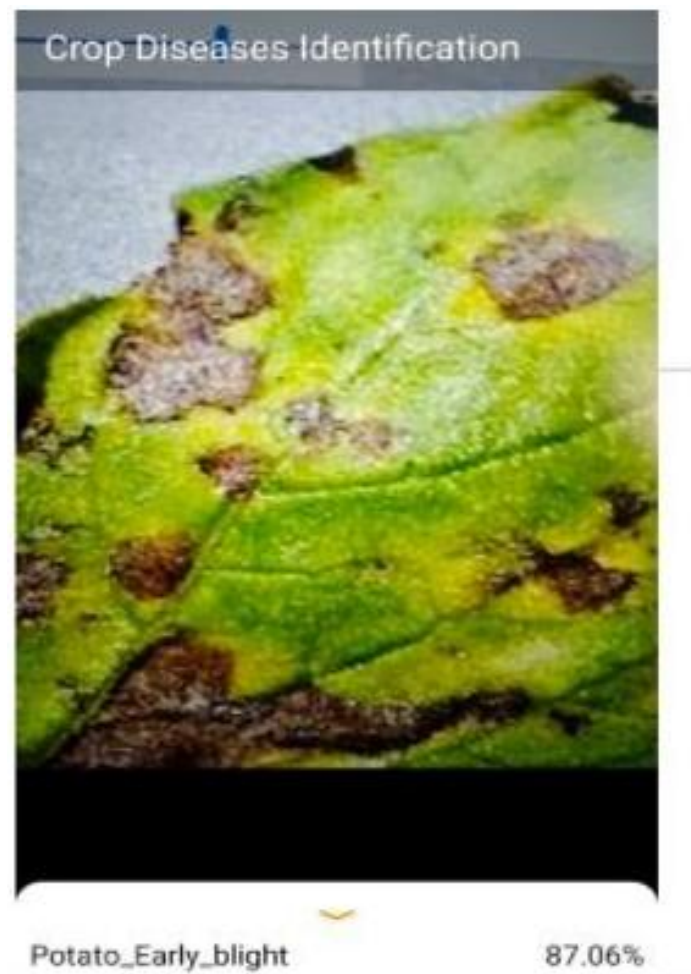

Fig. 7. Disease Predication Results

\section{CONCLUSION}

Protecting the integrity of the farmers is very important in a country where the primary occupation is Agriculture. This project and its implementation thus has made remarkable progress over the existing projects. The ability of crop disease prediction module to provide evidence about the cause of diseases due to deleterious fertilizers, Insecticides or inclement weather has been a huge problem in India for decades. Thus we have successfully used Artificial Intelligence in developing products that will aid the farmers in producing a better harvest.

\section{REFERENCES}

[1] "S. Pudumalar, E. Ramanujam, R. H. Rajashree, C. Kavya, T. Kiruthika and J. Nisha, "Crop recommendation system for pre- cision agriculture," 2016 Eighth International Conference on Ad- vanced Computing (ICoAC), Chennai, India, 2017, pp. 32-36, doi: 10.1109/ICoAC.2017.7951740.

[2] "Rohit Kumar Rajak, Ankit Pawar, Mitalee Pendke, Pooja Shinde, Suresh Rathod, Avinash Devare. "Crop Recommendation System to Maximize Crop Yield using Machine Learning Technique." International Research Journal of Engineering and Technology (IRJET) vol.4, issue 12, Dec 2017.

[3] G.T. Hariharan, G.P.S. Hariharan and R. Vijay Anandh,"Crop Disease Identification using Image Processing," International Journal of Latest Trends in Engineering and Technology (IJLTET) Vol. 6 Issue 4, March 2016.

[4] P.Sakthi and P.Yuvarani, "Detection and Removal of Weed between Crops in Agricultural Field using Image Processing", International Journal of Pure and Applied Mathematics Volume 118 No. 8 2018, 201-206

[5] V. Mehta, C. Jain, K. Kanchan and V. Sawant, quot;A Machine Learning Approach to Foretell the Probability of a Crop Contracting a Disease,quot; 2018 Fourth International Conference on Computing Communication Control and Automation (ICCUBEA), Pune, India, 2018, pp. 1-7, doi: 10.1109/ICCUBEA.2018.8697774.

[6] A. Savla, N. Israni, P. Dhawan, A. Mandholia, H. Bhadada and S. Bhardwaj, quot;Survey of classification algorithms for formulating yield prediction accuracy in precision agriculture,quot; 2015 International Conference on Innovations in Information, Embedded and Communica- tion Systems (ICIIECS), Coimbatore, 2015, pp. 1-7, doi: 10.1109/ICI- IECS.2015.7193120.

[7] Classification Algorithms - Random Forest, Oct. 2019, [Online]Available:https://www.tutorialspoint.com/machine/learning/withpython/machine-learning-with-pYthon-classification-algorithmsrandom-forest.html. 